\title{
Characterization of vaginal microbiota in Thai women
}

\author{
Auttawit Sirichoat $^{1}$, Pranom Buppasiri ${ }^{2}$, Chulapan Engchanil ${ }^{1}$, Wises Namwat ${ }^{1}$, Kiatichai Faksri $^{1}$, \\ Nipaporn Sankuntaw ${ }^{3}$, Ekawat Pasomsub ${ }^{4}$, Wasun Chantratita ${ }^{5}$, Viraphong Lulitanond ${ }^{\text {Corresp. } 1}$ \\ ${ }^{1}$ Department of Microbiology and Research and Diagnostic Center for Emerging Infectious Diseases, Faculty of Medicine, Khon Kaen University, Khon \\ Kaen, Thailand \\ 2 Department of Obstetrics and Gynecology, Faculty of Medicine, Khon Kaen University, Khon Kaen, Thailand \\ 3 Chulabhorn International College of Medicine, Thammasat University, Pathum Thani, Thailand \\ 4 Division of Virology, Department of Pathology, Faculty of Medicine Ramathibodi Hospital, Mahidol University, Bangkok, Thailand \\ 5 Medical Genome Center, Faculty of Medicine, Ramathibodi Hospital, Mahidol University, Bangkok, Thailand \\ Corresponding Author: Viraphong Lulitanond \\ Email address: viraphng@kku.ac.th
}

Background: The vaginal microbiota (VMB) plays a key role in women's reproductive health. VMB composition varies with ethnicity, making it necessary to characterize the VMB of the target population before interventions to maintain and/or improve the vaginal health are undertaken. Information on the VMB of Thai women is currently unavailable. We therefore characterized the VMB in normal Thai women. Methods: Vaginal samples derived from 25 Thai women were subjected to 16S rRNA gene next-generation sequencing (NGS) on the Ion Torrent PGM platform. Results: Two groups of VMB were detected, lactobacilli-dominated (LD) and non-lactobacilli dominated (NLD) groups. Lactobacillus iners was the most common species found in the LD group while Gardnerella vaginalis followed by Atopobium vaginae and Pseudumonas stutzeri were commonly found in the NLD group. Conclusions: The VMB patterns present in normal Thai women is essential information to further determine the factors associated with VMB patterns in vaginal health and disease and to develop proper management of reproductive health of Thai women. 


\section{Characterization of vaginal microbiota in Thai women}

2

3 Auttawit Sirichoat ${ }^{1}$, Pranom Buppasiri ${ }^{2}$, Chulapan Engchanil ${ }^{1}$, Wises Namwat ${ }^{1}$, Kiatichai

4 Faksri $^{1}$, Nipaporn Sankuntaw ${ }^{3}$, Ekawat Pasomsub ${ }^{4}$, Wasun Chantratita ${ }^{5}$, Viraphong Lulitanond ${ }^{1}$ 5

$6{ }^{1}$ Department of Microbiology and Research and Diagnostic Center for Emerging Infectious

7 Diseases, Faculty of Medicine, Khon Kaen University, Khon Kaen, 40002, Thailand.

$8{ }^{2}$ Department of Obstetrics and Gynecology, Faculty of Medicine, Khon Kaen University, Khon 9 Kaen, 40002, Thailand.

$10{ }^{3}$ Chulabhorn International College of Medicine, Thammasat University, Pathum Thani, 12120, 11 Thailand.

$12{ }^{4}$ Division of Virology, Department of Pathology, Faculty of Medicine Ramathibodi Hospital, 13 Mahidol University, Bangkok, 10400, Thailand.

$14{ }^{5}$ Medical Genome Center, Faculty of Medicine, Ramathibodi Hospital, Mahidol University, 15 Bangkok, 10400, Thailand.

17 Corresponding author:

18 Viraphong Lulitanond ${ }^{1}$

19 Department of Microbiology, Faculty of Medicine, Khon Kaen University, Khon Kaen, Thailand 2040002

21 E-mail: viraphng@kku.ac.th; viraphng@gmail.com 


\section{Abstract}

23 Background: The vaginal microbiota (VMB) plays a key role in women's reproductive health.

$24 \mathrm{VMB}$ composition varies with ethnicity, making it necessary to characterize the VMB of the

25 target population before interventions to maintain and/or improve the vaginal health are

26 undertaken. Information on the VMB of Thai women is currently unavailable. We therefore

27 characterized the VMB in normal Thai women.

28 Methods: Vaginal samples derived from 25 Thai women were subjected to 16S rRNA gene next-generation sequencing (NGS) on the Ion Torrent PGM platform.

Results: Two groups of VMB were detected, lactobacilli-dominated (LD) and non-lactobacilli dominated (NLD) groups. Lactobacillus iners was the most common species found in the LD group while Gardnerella vaginalis followed by Atopobium vaginae and Pseudumonas stutzeri were commonly found in the NLD group.

Conclusions: The VMB patterns present in normal Thai women is essential information to further determine the factors associated with VMB patterns in vaginal health and disease and to develop proper management of reproductive health of Thai women. 
40

41

42

43

44

45

46

47

48

49

\section{Introduction}

The vaginal microbiota (VMB) plays an important role in vaginal health and in particular it has a protective role against vaginal infection. The composition of the VMB varies greatly among ethnic groups (Ravel et al., 2011). Up to now there is no definite study about how the VMB varies by ethnicity, however, some studies suggested the differences in VMB by ethnicities may be related to host genetic variations, which associated with individual differences in immune response (Blekhman et al., 2015; Green et al., 2015; Ma et al., 2014). In addition, VMB composition can be affected by various internal and external factors, e.g. host physiology, transitional reproductive period, menstruation, pregnancy, infections, birth control procedures and amount of sexual activity (Eschenbach et al., 2000; Gajer et al., 2012; Huang et al., 2014; Romero et al., 2014; Smith \& Ravel, 2017; Vodstrcil et al., 2017). In reproductive-aged women, the VMB is dominated by various Lactobacillus species such as L. iners, L. crispatus, L. gasseri, and L. jensenii (Gajer et al., 2012; Ravel et al., 2011). In vitro studies found that Lactobacilli can inhibit pathogens through the production of hydrogen peroxide and lactic acid which could maintain an acidic environment in the vagina (Aroutcheva et al., 2001; Eschenbach et al., 1989).

Several studies have characterized the VMB in healthy, reproductive women of different ethnicities using DNA sequence-based methods, especially next-generation sequencing (NGS) techniques (Borgdorff et al., 2017; Fettweis et al., 2014; Ravel et al., 2011). Five community state types (CSTs) have been recognized based on bacterial relative abundance on the basis of 16S ribosomal RNA (rRNA) sequences obtained using NGS (Ravel et al., 2011; Zhou et al., 2004). Four of these CSTs, i.e. CST-I, CST-II, CST-III and CST-V, were dominated by $L$. crispatus, L. gasseri, L. iners and L. jensenii, respectively. An additional type, CST-IV, is comprised of a high diversity of strict and facultative anaerobic bacteria including members of 
63 the genera Gardnerella, Atopobium, Corynebacterium, Prevotella, Mobiluncus, Anaerococcus,

64 Sneathia and others, which are associated with vaginal infections especially bacterial vaginosis

65 (BV) (Fredricks et al., 2005; Ling et al., 2010; Ravel et al., 2011; Swidsinski et al., 2005). NGS-

66 based studies have previously explored the VMB in Asian, African and European women

67 (Borgdorff et al., 2017; Chaban et al., 2014; Fettweis et al., 2014; Ravel et al., 2011; van de

68 Wijgert et al., 2014). Among Asian populations, VMB in Chinese (Ling et al., 2013; Shi et al.,

69 2009), Japanese (Yoshimura et al., 2011; Zhou et al., 2010) and South Korean (Hong et al.,

70 2016; Lee et al., 2013) populations have already been studied, but women in South East Asia,

71 including Thailand, have not been investigated. To our knowledge, this is the first report to

72 characterize the VMB in healthy Thai women based on 16S rRNA gene sequences obtained

73 using NGS.

75 Materials and methods

76 Subject selection and sample collection

Twenty-five women attending the gynecologic out-patient clinics for cervical cancer screening, Srinagarind Hospital, Faculty of Medicine, Khon Kaen University, were enrolled in this study. The inclusion criteria used for subject selection specified non-pregnant women aged 20-45 years with regular menstruation, with normal vaginal examination and without any clinical symptoms on examination by one gynecologist. The exclusion criteria were women with the following conditions: having menstruation or sexual intercourse within the previous 24 hours, use of antibiotics or vaginal antimicrobials within the last month, presence of any intravaginal product, use of a vaginal douche in the past week, and active infection or diagnosis of any defined sexually transmitted disease. The subjects selected by the above criteria were defined as 
86

87

normal women. All participants provided written informed consent and filled out a questionnaire detailing their level of education, employment, sociodemographic characteristics, dietary habits, reproductive health habits, and sexual behaviors (including number of children), income per month, alcohol consumption per week, exercise per week, sexual activity per week, number of sexual partners in the past, regularity of their menstrual cycles in the last six months, current hormone contraceptive use, douching practice in the past month, abnormal vaginal discharge in the past month and female sterilization. This study was approved by the Ethics Committee of Khon Kaen University (HE601017).

A total of four points at mid right and left, anterior and posterior vaginal wall were swabbed with sterile cotton-tipped applicators by one gynecologist. The swabs were placed in sterile containers with phosphate-buffer saline (PBS) and stored at $-80^{\circ} \mathrm{C}$ until analysis.

\section{Genomic DNA extraction from vaginal swabs}

Genomic DNA was extracted using the method described by Ravel et al. (2011) with slight modification. In briefly, the vaginal swabs were thawed on ice and adhered material resuspended by vigorous vortexing for $5 \mathrm{~min}$. Bacterial cells were lysed by incubation for 1 hour at $37^{\circ} \mathrm{C}$ in TE50 buffer $(10 \mathrm{mM}$ Tris $\mathrm{HCl}, 50 \mathrm{mM}$ EDTA, $\mathrm{pH} 8.0)$ containing $10 \mathrm{mg} / \mathrm{mL}$ of lysozyme (Merck, Darmstadt, Germany), 25,000 U/mL of mutanolysin (Sigma Aldrich, USA, St. Louis) and 4,000 U/mL of lysostaphin (Sigma-Aldrich), followed by beating with $40-400 \mu \mathrm{M}$ glass beads (NucleoSpin ${ }^{\circledR}$ Bead Tubes Type B, Macherey-Nagel, Düren, Germany) at speed 10 for 2 min with air cooling using the Bullet Blender ${ }^{\circledR}$ Blue Homogenizer (BBX 24B, Next Advance, Inc., USA). After bead-beating, the crude lysates were processed for purification of genomic DNA using QIAamp ${ }^{\circledR}$ DNA Mini Kit (Qiagen, Hilden, Germany) according to the 
109

110

111

112

113

114

115

116

117

118

119

120

121

122

123

124

125

126

127

128

129

130

131

manufacturer's recommendations. Integrity, size and concentration of the purified genomic DNA was determined using Fragment Analyzer ${ }^{\mathrm{TM}}$ (Advanced Analytical Technologies, Inc., USA).

\section{S rRNA gene sequencing by NGS}

16S rRNA gene sequencing was done as previously described (Barb et al., 2016) with minor modifications. The seven $16 \mathrm{~S}$ hypervariable regions were amplified with 2 primer sets, one set for amplifying the V2, V4, V8 hypervariable regions and the other set for amplifying the V3, V6-7, V9 hypervariable regions. Primers were supplied with the Ion $16 \mathrm{~S}^{\text {TM }}$ Metagenomics Kit (Life Technologies, Grand Island, NY) and used according to the manufacturer's recommendations. The combined PCR products were processed to make the DNA library by using the Ion Plus ${ }^{\mathrm{TM}}$ Fragment Library Kit (Life Technologies) with sample indexing using the Ion Xpress ${ }^{\mathrm{TM}}$ Barcodes Adapters 1-32 Kit (Life Technologies). The adapter-ligated and nickrepaired DNA was amplified with the following steps: 1 hold at $95^{\circ} \mathrm{C}$ for $5 \mathrm{~min}$; 5-7 cycles of $95^{\circ} \mathrm{C}$ for $15 \mathrm{sec}, 58^{\circ} \mathrm{C}$ for $15 \mathrm{sec}, 70^{\circ} \mathrm{C}$ for $1 \mathrm{~min}$; hold at $4^{\circ} \mathrm{C}$. The PCR products were then purified using Agencourt ${ }^{\circledR}$ AMPure ${ }^{\circledR}$ XP Reagent (Beckman Coulter, Inc, Atlanta, Georgia). The processed libraries were quantified using a Bioanalyzer ${ }^{\circledR}$ instrument (Agilent Technologies, Santa Clara, CA.) with the use of the Agilent ${ }^{\circledR}$ High-Sensitivity DNA Kit. Equal volumes of all 25 samples were pooled and processed with the Ion PGM ${ }^{\mathrm{TM}} \mathrm{Hi}-\mathrm{Q}^{\mathrm{TM}} \mathrm{OT} 2 \mathrm{Kit}$ in the Ion OneTouch $^{\mathrm{TM}} 2$ System (Life Technologies) according to the manufacturer' instructions.

Sequencing was performed in an Ion $318^{\mathrm{TM}}$ Chip (Life Technologies) using the Ion PGM ${ }^{\mathrm{TM}}$ Sequencing 400 Kit on the Ion PGM ${ }^{\text {TM }}$ System (Life Technologies).

\section{Sequence data analysis}



version 5.0.5 (Life Technologies) with default parameters. All sequences were trimmed according to the quality. To pass, a sequence read had a following criteria: 1) a perfect match to a barcode sequence and the primer; 2) was at least $200 \mathrm{bp}$ in length; 3) had no more than two undetermined bases; and 4) had an average quality score greater than Q20. Sequences were analyzed using Ion Reporter ${ }^{\mathrm{TM}}$ Software version 5.6 with the $16 \mathrm{~S}$ Metagenomics workflow module (Life Technologies). Clustering of operational taxonomic units (OTUs) and taxonomic classification were performed based on two-step Basic Local Alignment Search Tool (BLAST) that maps to two separate reference libraries. In the first step, reads were aligned to the MicroSEQ $^{\circledR} 16 \mathrm{~S}$ Reference Library v2013.1 database (Woo et al., 2003). Subsequently, any unaligned reads subject to second alignment to the Greengenes v13.5 database (DeSantis et al., 2006). At least 10 of unique reads were valid and $\geq 90 \%$ for the alignment coverage needed between hit and query. Genus- and species-level identifications were accepted at $\geq 97 \%$ and $\geq 99 \%$ sequence identity, respectively (Drancourt et al., 2000). In case of any unusual microorganism, the sequence reads were reanalyzed by BLAST with the National Center for Biotechnology Information (NCBI) taxonomy database. Any OTUs represented by only one sequence (singletons) and those with fewer than 10 reads in the sample were excluded from further analysis. Alpha- (within sample) and beta- (among samples) diversities were calculated using Quantitative Insights Into Microbial Ecology (QIIME) software (Caporaso et al., 2010). Alpha diversity metrics, consisting of the Shannon diversity index, observed species and Chao 1 index, were graphed using GraphPad Prism version 5.01 (GraphPad Software Inc., USA). The beta diversity metric was calculated according to Bray-Curtis dissimilarity index (Bray \& Curtis,

154 1957) and displayed through a principal coordinates analysis (PCoA) plot. Heat map and 
155 hierarchical clustering tree were generated using R Studio (Version 1.1.414, Boston, USA)

156 employing R version 3.4.1 (R Development Core Team). Raw sequences were deposited in the

157 NCBI Sequence Read Archive (SRA) accession number SRP158176.

158

159

\section{Statistical analysis}

160

Statistical analysis was performed using IBM SPSS Statistics for Windows, version 19.0

161 software (SPSS Inc., Chicago, IL, USA). Demographic characteristics between two groups were

162 compared using the chi-squared or Fisher's exact test. Mann-Whitney U test for non-parametric 163 data was used for bacterial abundance and diversity comparisons between two groups. $P$-values 164 less than 0.05 were considered as statistically significant.

\section{Results}

167

168

169

170

171

172

173

174

175

176

177

\section{Participant characteristics}

The median age and body mass index of the enrolled subjects were 39 years (ranging from 31 to 45 ) and $21.2 \mathrm{~kg} / \mathrm{m}^{2}$ (ranging from 16.0 to 35.8), respectively. Subjects fell into two groups: lactobacilli-dominated (LD) and non-lactobacilli dominated (NLD) groups (see below).

No significant association between sociodemographic characteristics and VMB group (LD or NLD) was found, as shown in Table 1.

\section{Analysis of VMB in the studied subjects}

High-throughput sequencing data of all vaginal samples derived from Ion Torrent PGM yielded a total of 4,775,332 raw sequences with an average of 191,013 reads per sample (ranging from 33,969 to 382,574 reads). We discarded any reads shorter than 150 bp and those OTUs represented fewer than 10 times in the sample. This left a total of 4,189,265 valid reads with an 
178 average of 167,570 reads per sample (ranging from 28,825 to 333,480 reads) for analysis. The

179 average length of included reads was $243 \mathrm{bp}$ (ranging from 237 to $253 \mathrm{bp}$ ). A total of 3,553,612

180 mapped reads were clustered into OTUs and given taxonomic identification. Forty-one genera

181 and 72 species were identified (Table S1 and S2). VMB were clustered into two distinct groups:

182 LD ( $n=14)$ and NLD ( $=11)$. In the LD group, the average of relative abundance of Lactobacillus

183 species is $90 \%$ and the relative abundance of this species among the LD individuals is $\geq 60 \%$ and

184 could be further divided into two subgroups: one dominated by L. iners $(\mathrm{n}=10)$ and the second

185 subgroup ( $\mathrm{n}=4$ ) was dominated by L. crispatus, L. gasseri, L. jensenii and L. johnsonii. The NLD

186 group could similarly be divided into two subgroups: one dominated by Gardnerella vaginalis

$187(n=3)$ and the other $(n=8)$ dominated by anaerobic bacteria of a mixture of several species

188 including G. vaginalis, Atopobium vaginae and Pseudomonas stutzeri. Fig. 1 represents the

189 hierarchical clustering tree (Fig. 1A) and the heat map (Fig. 1B) of bacterial taxa identified from

19025 vaginal samples. Table S3 indicates the mean percentage representation of each bacterial

191 genus present in these two groups.

In this study, alpha-diversity for determination of bacterial diversity within individual

193

194

195

196

197

198

199

200

women used three metrics: Shannon diversity index (estimated evenness and richness), observed species (observed bacterial richness) and Chaol (estimated bacterial richness). The Shannon diversity index differed significantly between the two groups $(P<0.001)$ (Fig. 2$)$. The observed and estimated numbers of OTUs in individuals in the NLD group were significantly higher than those in the LD group (both $P<0.001$ ) (Fig. 3). All these results indicated that the bacterial communities in NLD individuals have more species diversity than those in LD individuals. In beta-diversity analysis, the PCoA plot based on taxonomic profiles showed that some distinct clustering appeared to overlap between two groups (Fig. 4). This result suggested that the VMB 
201 of both LD and NLD individuals did not tightly cluster by group. In addition, the relative

202 distances based on the Bray-Curtis dissimilarity index showed significantly greater distances

203 among LD individual samples than among NLD individual samples $(0.82 \pm 0.10$ versus

$2040.72 \pm 0.09, P=0.021)$. Since the distance measurement indicates the degree of similarity among

205 samples (van de Wijgert \& Jespers, 2016), this result indicates that VMB in LD individuals

206 resemble one another more closely than do those in NLD individuals.

207 The number of species unique to either LD or NLD, or common to both groups, were 14

208 (19.44\%), 41 (56.95\%) and 17 (23.61\%), respectively (Fig. 5). The 17 common bacterial species

209 found in both groups were A. vaginae, Finegoldia magna, G. vaginalis, L. crispatus, L. gasseri,

210 L. iners, L. johnsonii, L. kefiranofaciens, L. vaginalis, Megasphaera spp., Peptoniphilus harei,

211 Peptostreptococcus anaerobius, Prevotella bivia, P. timonensis, Pseudomonas stutzeri,

212 Streptococcus intermedius and Ureaplasma parvum. Fig. 6 represents the most abundant

213 bacterial species detected in the vaginal samples of LD and NLD groups. G. vaginalis, $A$.

214 vaginae and $P$. bivia were significantly associated with the NLD group $(P<0.05)$ while $L$. iners

215 was significantly associated with the LD group $(P<0.05)$. The bacterial species with abundance

216 less than 1\% are F. magna, L. kefiranofaciens, L. vaginalis, Peptoniphilus harei,

217 Peptostreptococcus anaerobius, S. intermedius and U. parvum.

\section{Discussion}

The community composition of the VMB can vary greatly among women. However,

221 although it can be absent entirely, Lactobacillus is likely to be the dominant, and sometimes

222 only, genus in VMB (Cherpes et al., 2008b; Linhares et al., 2011; Ma et al., 2012; Petrova et

223 al., 2015; Smith \& Ravel, 2017). We found the VMB of normal Thai women to fall into two 
224 groups. In the LD group, lactobacilli, and especially L. iners, can make up $90 \%$ of the bacterial 225 cells present. In the NLD group, G. vaginalis followed by A. vaginae and Pseudomonas stutzeri 226 jointly constitute the majority of bacteria present. Previous studies found the difference on the 227 composition of VMB among reproductive age women of different ethnicity (Fettweis et al.,

228

2014; Ravel et al., 2011). Studied on VMB of asymptomatic North America women by Ravel et al. (2011) using Roche 454 sequencing (454 sequencing) of the V1-V2 regions of 16S rRNA gene found that Lactobacillus-dominated (80-90\%) is the most common type of VMB in Asian and White women but less common (approx.. 60\%) in Hispanic and African American women. Furthermore, a Lactobacillus-dominated VMB was also found in normal Chinese and Korean women by using 454 sequencing of the V3 and V3-V5 regions, respectively (Hong et al., 2016; Ling et al., 2010). A high abundance of Lactobacillus species is generally considered as a good biomarker for a healthy vaginal ecosystem (Petrova et al., 2015). Lactobacilli promote a protective environment in the vagina by several mechanisms, such as production of hydrogen peroxide, bacteriocins or certain metabolites, that can inhibit the colonization and growth of various vaginal pathogens (Aroutcheva et al., 2001; Eschenbach et al., 1989).

Lactobacillus iners, the dominant species of VMB in our study, is also the most common species in asymptomatic North American women with Asian ethnicity and in African American women by using 454 sequencing of the V1-V2 and V1-V3 regions of 16S rRNA genes, respectively (Fettweis et al., 2014; Ravel et al., 2011). Previous studies with different sequencing platforms of 16S rRNA gene, e.g. Illumina (V3-V4) and 454 (V1-V2 or V1-V3) sequencing, found other Lactobacillus species such as L. crispatus, L. gasseri and L. jensenii are commonly the dominant species in White or European women, but are less common in Asian, Black and Hispanic women (Borgdorff et al., 2017; Fettweis et al., 2014; Ravel et al., 2011). The 
247 difference in VMB composition likely have a genetic and ethnic basis (Green et al., 2015).

248 Nevertheless, a systematic review by van de Wijgert et al. (2014) found that L. iners is present in

249 most women, either healthy or with BV, while L. crispatus is commonly found only in healthy

250 women. Longitudinal studies have found that women who have L. iners-dominated VMB at

251 baseline are more likely to transition to a BV-associated VMB than women who have $L$.

252 crispatus-dominated VMB. Lactobacillus crispatus-dominated VMB more often transitions to an

253 L. iners-dominated or mixed lactobacilli VMB than to a BV-associated VMB (Gajer et al.,

254 2012). The role of $L$. iners remains controversial. It appears that $L$. iners is well adapted to the

255 vaginal niche, able to survive in a BV-like environment and often persisting after antibiotic

256 treatment (Huang et al., 2014; van de Wijgert et al., 2014). It may help to restore a lactobacilli-

257 dominated microbiota during and after dysbiosis and/or after antibiotic treatment (van de Wijgert

258 et al., 2014). Thus, L. iners-dominated VMB might indicate a transitional condition between

259 normal and abnormal. However, since our participants with L. iners-dominated VMB show no

260 symptom both before and after sample collection perhaps this is "normal" for Thai women.

261 Other studies have proposed that genetic/ethnic differences in immune responses might make the

262 vaginal mucosa more favorable for colonization by L. iners over L. crispatus in African women

263 (Doh et al., 2004; Nguyen et al., 2004; Ryckman et al., 2008). However, the exact mechanism

264 leading to transitions in the microbiota are not known and might be affected by various factors,

265 e.g. the hormonal changes, sexual intercourse, smoking, personal hygiene, antibiotic treatment,

266 ethnicity, individual differences in immune status/response, vaginal epithelial cells and their

267 secretions (Petrova et al., 2015; Petrova et al., 2017; van de Wijgert et al., 2014).

Nearly half of the subjects (11 of the 25 subjects) was dominated by a suite of species,

not by a single taxon. This type of VMB was previously found in $40.4 \%, 19.8 \%$ and $38.5 \%$ of 
270

271

272

274

275

276

277

asymptomatic Black or African women (Ravel et al., 2011), Asian women (Ravel et al., 2011), and women in Amsterdam (Borgdorff et al., 2017), respectively. Previous systematic reviews have revealed that some healthy women have this type of VMB (Huang et al., 2014; Petrova et al., 2015), which is dominated by members of the genera Gardnerella, Atopobium, Prevotella, Corynebacterium, Anaerococcus, Peptoniphilus, Mobiluncus and Sneathia. These species have evolved mechanisms to persist in a slightly alkaline environment and to adhere to the vaginal epithelial cells. It has been suggested that such a suite of bacteria is able to maintain the protective function of the vagina through lactic acid production to lower vaginal $\mathrm{pH}$ (Gajer et al., 2012). Lactic acid can be produced by either homolactic or heterolactic acid fermentation by certain facultatively or strictly anaerobic bacteria such as Atopobium, Streptococcus, Staphylococcus, Megashara and Leptotrichia (Petrova et al., 2015; Zhou et al., 2004). These bacteria might contribute to maintain the balance of the healthy vaginal ecosystem. Thus, the polymicrobial VMB seen in our and other studies indicated that this type of VMB can be present in normal healthy women (Petrova et al., 2015).

It should be noted that in the study of VMB using $16 \mathrm{~S}$ rRNA gene sequencing there are difference in the usage of $16 \mathrm{~S}$ rRNA region to generate reads and in the database for taxonomic classification among the studies. There is much speculation on which hypervariable region provides sufficient sequences diversity to identify the most bacteria accurately (Liu et al., 2008; Schloss et al., 2011). Many studies found that using different hypervariable regions of the gene provides variable results (Barb et al., 2016; Chakravorty et al., 2007; Youssef et al., 2009). The best solution would be to sequence the entire $16 \mathrm{~S}$ rRNA gene; however, this approach is not possible with short read NGS platforms. In this study, we used Ion Torrent PGM platform which using multiple variable regions (V2-V4 and V6-V9 regions) to explore the VMB which would 
293 provide sufficient sequence to identify the most bacteria accurately. In addition, in the taxonomic

294 classification in this study primarily performed by alignment to the MicroSEQ ${ }^{\circledR} 16 \mathrm{~S}$ Reference

295 Library v2013.1 database and the Greengenes v13.5 database as described in Materials and

296 Methods above revealed some unusual microorganisms, i.e. Olsenella umbonata and O. profusa.

297 The sequence reads of these two microorganisms were reanalyzed with NCBI database and

298 found them to belong to the most common species, i.e. A. vaginae (\% identity of $O$. umbonata

299 and $A$. vaginae are 99 and 100, respectively, and the identity of both O. profusa and A. vaginae is

$30099 \%$ ). For the other microorganisms, the classification using either MicroSEQ ${ }^{\circledR} 16 \mathrm{~S}$ Reference

301 Library/Greengenes database or NCBI database found to give similar results.

Previous studies suggested that differences in bacterial composition in VMB might be

303

304

305

306

307

308

309

310

311

312

313

314

315

associated with both intrinsic and extrinsic factors, e.g. ethnicity, sociodemography,

environment, pregnancy, smoking status, sexual behavior, number of sexual partners, alcohol consumption and host genetic factors (Borgdorff et al., 2017; Fettweis et al., 2014; Huang et al., 2014). Five different VMB clusters were described among four different ethnic groups (White, Black, Hispanic and Asian women) in North America (Ravel et al., 2011). Two additional clusters, dominated by G. vaginalis or BV-associated bacteria (BVAB) type 1, were found in another study in African American women and women of European ancestry (Fettweis et al., 2014). Genomic markers have revealed that differences in VMB by ethnicity might be related to mitochondrial DNA (mtDNA) polymorphisms (Blekhman et al., 2015; Green et al., 2015; Ma et al., 2014). High estradiol during pregnancy has been reported to be associated with high levels of lactobacilli and low bacterial diversity (Aagaard et al., 2012; Petricevic et al., 2012), and with low incidence of BV and low prevalence of BVAB during pregnancy (Romero et al., 2014). Smoking is a risk factor of BV (Cherpes et al., 2008a; Ryckman et al., 2009) and smokers appear 
316 to have a lower proportion of vaginal Lactobacillus spp. than do non-smokers (Brotman et al.,

317 2014). Sexual behaviors and number of sexual partners have been implicated the bacterial

318 composition and diversity of the VMB (Huang et al., 2014; van de Wijgert et al., 2014). Prior

319 studies have shown that the bacterial diversity in women engaging in high-risk sexual behavior

320 was increased along with the decline of lactobacilli (Wessels et al., 2017). Lastly, due to a small

321 sample size in this study, the large sample size in future study is inevitably required to draw a

322 more meaningful conclusion.

323

324

\section{Conclusion}

Our study is the first to report the types of VMB in normal Thai women using 16S rRNA gene sequence data obtained by NGS. Two major groups were recognized, i.e. LD and NLD

327 groups. Lactobacillus iners is the dominant species of VMB in the LD group while several species are abundant in VMB in the NLD group. The information on VMB in Thai women is a starting point for further studying of factors involved in the development and maintenance of VMB communities in vaginal health and disease. A better understanding of VMB, including their interaction with external and internal factors, will assist in development of effective strategies to manage reproductive health of Thai women.

\section{Acknowledgements}

We would like to acknowledge Professor David Blair for editing the manuscript via Publication 336 Clinic KKU, Thailand. 


\section{References}

338 Aagaard K, Riehle K, Ma J, Segata N, Mistretta TA, Coarfa C, Raza S, Rosenbaum S, Van den Veyver I, Milosavljevic A, Gevers D, Huttenhower C, Petrosino J, and Versalovic J. 2012. A metagenomic approach to characterization of the vaginal microbiome signature in pregnancy. PLoS One 7:e36466 DOI: 10.1371/journal.pone.0036466.

Aroutcheva A, Gariti D, Simon M, Shott S, Faro J, Simoes JA, Gurguis A, and Faro S. 2001. Defense factors of vaginal lactobacilli. American Journal of Obstetrics and Gynecology

Barb JJ, Oler AJ, Kim HS, Chalmers N, Wallen GR, Cashion A, Munson PJ, and Ames NJ. 2016. Development of an Analysis Pipeline Characterizing Multiple Hypervariable Regions of 16S rRNA Using Mock Samples. PLoS One 11:e0148047 DOI: 10.1371/journal.pone.0148047.

Blekhman R, Goodrich JK, Huang K, Sun Q, Bukowski R, Bell JT, Spector TD, Keinan A, Ley RE, Gevers D, and Clark AG. 2015. Host genetic variation impacts microbiome composition across human body sites. Genome Biology 16:191 DOI: 10.1186/s13059015-0759-1.

Borgdorff H, van der Veer C, van Houdt R, Alberts CJ, de Vries HJ, Bruisten SM, Snijder MB, Prins M, Geerlings SE, Schim van der Loeff MF, and van de Wijgert J. 2017. The association between ethnicity and vaginal microbiota composition in Amsterdam, the Netherlands. PLoS One 12:e181135 DOI: 10.1371/journal.pone.0181135.

Bray JR, and Curtis JT. 1957. An Ordination of the Upland Forest Communities of Southern Wisconsin. Ecological Monographs 27:325-349 DOI: 10.2307/1942268. 
359 Brotman RM, He X, Gajer P, Fadrosh D, Sharma E, Mongodin EF, Ravel J, Glover ED, and

360 Rath JM. 2014. Association between cigarette smoking and the vaginal microbiota: a

361 pilot study. BMC Infectious Diseases 14:471 DOI: 10.1186/1471-2334-14-471.

362 Caporaso JG, Kuczynski J, Stombaugh J, Bittinger K, Bushman FD, Costello EK, Fierer N, Pena AG, Goodrich JK, Gordon JI, Huttley GA, Kelley ST, Knights D, Koenig JE, Ley RE, Lozupone CA, McDonald D, Muegge BD, Pirrung M, Reeder J, Sevinsky JR, Turnbaugh PJ, Walters WA, Widmann J, Yatsunenko T, Zaneveld J, and Knight R. 2010. QIIME allows analysis of high-throughput community sequencing data. Nature Methods 7:335-

Chaban B, Links MG, Jayaprakash TP, Wagner EC, Bourque DK, Lohn Z, Albert AY, van Schalkwyk J, Reid G, Hemmingsen SM, Hill JE, and Money DM. 2014. Characterization of the vaginal microbiota of healthy Canadian women through the menstrual cycle. Microbiome 2:23 DOI: 10.1186/2049-2618-2-23. Microbiological Methods 69:330-339 DOI: 10.1016/j.mimet.2007.02.005. factors for acquisition of bacterial vaginosis include sexual activity, absence of hydrogen peroxide-producing lactobacilli, black race, and positive herpes simplex virus type 2 serology. Sexually Transmitted Diseases 35:78-83 DOI: 10.1097/OLQ.0b013e318156a5d0. 
380

381

382

383

384

385

386

387

388

389

390

391

392

393

394

395

396

397

398

399

400

401

402

Cherpes TL, Marrazzo JM, Cosentino LA, Meyn LA, Murray PJ, and Hillier SL. 2008 b.

Hormonal contraceptive use modulates the local inflammatory response to bacterial vaginosis. Sexually Transmitted Infections 84:57-61 DOI: 10.1136/sti.2007.026625.

DeSantis TZ, Hugenholtz P, Larsen N, Rojas M, Brodie EL, Keller K, Huber T, Dalevi D, Hu P, and Andersen GL. 2006. Greengenes, a chimera-checked 16S rRNA gene database and workbench compatible with ARB. Applied and Environmental Microbiology 72:50695072 DOI:10.1128/AEM.03006-05.

Doh K, Barton PT, Korneeva I, Perni SC, Bongiovanni AM, Tuttle SL, Skupski DW, and Witkin SS. 2004. Differential vaginal expression of interleukin-1 system cytokines in the presence of Mycoplasma hominis and Ureaplasma urealyticum in pregnant women. Infectious Diseases in Obstetrics and Gynecology 12:79-85 DOI: $10.1080 / 10647440400003667$.

Drancourt M, Bollet C, Carlioz A, Martelin R, Gayral JP, and Raoult D. 2000. 16S ribosomal DNA sequence analysis of a large collection of environmental and clinical unidentifiable bacterial isolates. Journal of Clinical Microbiology 38:3623-3630.

Eschenbach DA, Davick PR, Williams BL, Klebanoff SJ, Young-Smith K, Critchlow CM, and Holmes KK. 1989. Prevalence of hydrogen peroxide-producing Lactobacillus species in normal women and women with bacterial vaginosis. Journal of Clinical Microbiology $27: 251-256$.

Eschenbach DA, Thwin SS, Patton DL, Hooton TM, Stapleton AE, Agnew K, Winter C, Meier A, and Stamm WE. 2000. Influence of the normal menstrual cycle on vaginal tissue, discharge, and microflora. Clinical infectious diseases : an official publication of the Infectious Diseases Society of America 30:901-907 DOI: 10.1086/313818. 
403 Fettweis JM, Brooks JP, Serrano MG, Sheth NU, Girerd PH, Edwards DJ, Strauss JF, 3rd, 404 Vaginal Microbiome C, Jefferson KK, and Buck GA. 2014. Differences in vaginal 405 406 microbiome in African American women versus women of European ancestry. Microbiology 160:2272-2282 DOI: 10.1099/mic.0.081034-0.

407 408 409 410 411
Fredricks DN, Fiedler TL, and Marrazzo JM. 2005. Molecular identification of bacteria associated with bacterial vaginosis. The New England journal of medicine 353:1899-1911 DOI: $10.1056 /$ NEJMoa043802.

Gajer P, Brotman RM, Bai G, Sakamoto J, Schutte UM, Zhong X, Koenig SS, Fu L, Ma ZS, Zhou X, Abdo Z, Forney LJ, and Ravel J. 2012. Temporal dynamics of the human vaginal microbiota. Science Translational Medicine 4:132ra152 DOI: 10.1126/scitranslmed.3003605.

Green KA, Zarek SM, and Catherino WH. 2015. Gynecologic health and disease in relation to the microbiome of the female reproductive tract. Fertility and Sterility 104:1351-1357 DOI: 10.1016/j.fertnstert.2015.10.010.

Hong KH, Hong SK, Cho SI, Ra E, Han KH, Kang SB, Kim EC, Park SS, and Seong MW. 2016. Analysis of the Vaginal Microbiome by Next-Generation Sequencing and Evaluation of its Performance as a Clinical Diagnostic Tool in Vaginitis. Annals of Laboratory Medicine 36:441-449 DOI: 10.3343/alm.2016.36.5.441.

Huang B, Fettweis JM, Brooks JP, Jefferson KK, and Buck GA. 2014. The changing landscape of the vaginal microbiome. Clinics in Laboratory Medicine 34:747-761 DOI: 10.1016/j.cll.2014.08.006. 
424 Lee JE, Lee S, Lee H, Song YM, Lee K, Han MJ, Sung J, and Ko G. 2013. Association of the

425

426

427

428

429

430

431

432

433

434

435

436

437

438

439

440

441

442

443

444

445

vaginal microbiota with human papillomavirus infection in a Korean twin cohort. PLoS One 8:e63514 DOI: 10.1371/journal.pone.0063514.

Ling Z, Kong J, Liu F, Zhu H, Chen X, Wang Y, Li L, Nelson KE, Xia Y, and Xiang C. 2010. Molecular analysis of the diversity of vaginal microbiota associated with bacterial vaginosis. BMC Genomics 11:488 DOI: 10.1186/1471-2164-11-488.

Ling Z, Liu X, Luo Y, Wu X, Yuan L, Tong X, Li L, and Xiang C. 2013. Associations between vaginal pathogenic community and bacterial vaginosis in Chinese reproductive-age women. PLoS One 8:e76589 DOI: 10.1371/journal.pone.0076589.

Linhares IM, Summers PR, Larsen B, Giraldo PC, and Witkin SS. 2011. Contemporary perspectives on vaginal $\mathrm{pH}$ and lactobacilli. American Journal of Obstetrics and Gynecology 204:120 e121-125 DOI: 10.1016/j.ajog.2010.07.010.

Liu Z, DeSantis TZ, Andersen GL, and Knight R. 2008. Accurate taxonomy assignments from 16S rRNA sequences produced by highly parallel pyrosequencers. Nucleic Acids Research 36:e120 DOI: 10.1093/nar/gkn491.

Ma B, Forney LJ, and Ravel J. 2012. Vaginal microbiome: rethinking health and disease. Annual Review of Microbiology 66:371-389 DOI: 10.1146/annurev-micro-092611-150157.

Ma J, Coarfa C, Qin X, Bonnen PE, Milosavljevic A, Versalovic J, and Aagaard K. 2014. mtDNA haplogroup and single nucleotide polymorphisms structure human microbiome communities. BMC Genomics 15:257 DOI: 10.1186/1471-2164-15-257.

Nguyen DP, Genc M, Vardhana S, Babula O, Onderdonk A, and Witkin SS. 2004. Ethnic differences of polymorphisms in cytokine and innate immune system genes in pregnant 
women. Obstetrics and Gynecology 104:293-300 DOI:

447 10.1097/01.AOG.0000133486.85400.5e.

448

449

450

451

452

453

454

455

456

457

458

459

460

461

462

463

464

465

466

467

468

Petricevic L, Domig KJ, Nierscher FJ, Krondorfer I, Janitschek C, Kneifel W, and Kiss H. 2012. Characterisation of the oral, vaginal and rectal Lactobacillus flora in healthy pregnant and postmenopausal women. European Journal of Obstetrics, Gynecology, and Reproductive Biology 160:93-99 DOI: 10.1016/j.ejogrb.2011.10.002.

Petrova MI, Lievens E, Malik S, Imholz N, and Lebeer S. 2015. Lactobacillus species as biomarkers and agents that can promote various aspects of vaginal health. Frontiers in Physiology 6:81 DOI: 10.3389/fphys.2015.00081.

Petrova MI, Reid G, Vaneechoutte M, and Lebeer S. 2017. Lactobacillus iners: Friend or Foe? Trends in Microbiology 25:182-191 DOI: 10.1016/j.tim.2016.11.007.

Ravel J, Gajer P, Abdo Z, Schneider GM, Koenig SS, McCulle SL, Karlebach S, Gorle R, Russell J, Tacket CO, Brotman RM, Davis CC, Ault K, Peralta L, and Forney LJ. 2011. Vaginal microbiome of reproductive-age women. Proceedings of the National Academy of Sciences of the United States of America 108 Suppl 1:4680-4687 DOI: 10.1073/pnas.1002611107.

Romero R, Hassan SS, Gajer P, Tarca AL, Fadrosh DW, Nikita L, Galuppi M, Lamont RF, Chaemsaithong P, Miranda J, Chaiworapongsa T, and Ravel J. 2014. The composition and stability of the vaginal microbiota of normal pregnant women is different from that of non-pregnant women. Microbiome 2:4 DOI: 10.1186/2049-2618-2-4.

Ryckman KK, Simhan HN, Krohn MA, and Williams SM. 2009. Predicting risk of bacterial vaginosis: the role of race, smoking and corticotropin-releasing hormone-related genes. Molecular Human Reproduction 15:131-137 DOI: 10.1093/molehr/gan081. 
469 Ryckman KK, Williams SM, Krohn MA, and Simhan HN. 2008. Racial differences in cervical

470

471

472

473

474

475

476

477

478

479

480

481

482

483

484

485

486

487

488

489

490

491 cytokine concentrations between pregnant women with and without bacterial vaginosis. Journal of Reproductive Immunology 78:166-171 DOI: 10.1016/j.jri.2008.01.003.

Schloss PD, Gevers D, and Westcott SL. 2011. Reducing the effects of PCR amplification and sequencing artifacts on 16S rRNA-based studies. PLoS One 6:e27310 DOI: 10.1371/journal.pone.0027310.

Shi Y, Chen L, Tong J, and Xu C. 2009. Preliminary characterization of vaginal microbiota in healthy Chinese women using cultivation-independent methods. The Journal of Obstetrics and Gynaecology Research 35:525-532 DOI: 10.1111/j.14470756.2008.00971.x.

Smith SB, and Ravel J. 2017. The vaginal microbiota, host defence and reproductive physiology. The Journal of Physiology 595:451-463 DOI: 10.1113/JP271694.

Swidsinski A, Mendling W, Loening-Baucke V, Ladhoff A, Swidsinski S, Hale LP, and Lochs H. 2005. Adherent biofilms in bacterial vaginosis. Obstetrics and Gynecology 106:10131023 DOI: 10.1097/01.AOG.0000183594.45524.d2.

van de Wijgert JH, Borgdorff H, Verhelst R, Crucitti T, Francis S, Verstraelen H, and Jespers V. 2014. The vaginal microbiota: what have we learned after a decade of molecular characterization? PLoS One 9:e105998 DOI: 10.1371/journal.pone.0105998.

van de Wijgert JH, and Jespers V. 2016. Incorporating microbiota data into epidemiologic models: examples from vaginal microbiota research. Annals of Epidemiology 26:360-365 DOI: 10.1016/j.annepidem.2016.03.004.

Vodstrcil LA, Twin J, Garland SM, Fairley CK, Hocking JS, Law MG, Plummer EL, Fethers KA, Chow EP, Tabrizi SN, and Bradshaw CS. 2017. The influence of sexual activity on 
492

493

494

495

496

497

498

499

500

501

502

503

504

505

506

507

508

509

510

511

512

513

514

the vaginal microbiota and Gardnerella vaginalis clade diversity in young women. PLoS One 12:e171856 DOI: 10.1371/journal.pone.0171856.

Wessels JM, Lajoie J, Vitali D, Omollo K, Kimani J, Oyugi J, Cheruiyot J, Kimani M, Mungai JN, Akolo M, Stearns JC, Surette MG, Fowke KR, and Kaushic C. 2017. Association of high-risk sexual behaviour with diversity of the vaginal microbiota and abundance of Lactobacillus. PLoS One 12:e187612 DOI: 10.1371/journal.pone.0187612.

Woo PC, Ng KH, Lau SK, Yip KT, Fung AM, Leung KW, Tam DM, Que TL, and Yuen KY. 2003. Usefulness of the MicroSeq 500 16S ribosomal DNA-based bacterial identification system for identification of clinically significant bacterial isolates with ambiguous biochemical profiles. Journal of Clinical Microbiology 41:1996-2001.

Yoshimura K, Morotomi N, Fukuda K, Nakano M, Kashimura M, Hachisuga T, and Taniguchi H. 2011. Intravaginal microbial flora by the 16S rRNA gene sequencing. American Journal of Obstetrics and Gynecology 205:235 e231-239 DOI: 10.1016/j.ajog.2011.04.018.

Youssef N, Sheik CS, Krumholz LR, Najar FZ, Roe BA, and Elshahed MS. 2009. Comparison of species richness estimates obtained using nearly complete fragments and simulated pyrosequencing-generated fragments in 16S rRNA gene-based environmental surveys. Applied and Environmental Microbiology 75:5227-5236 DOI: 10.1128/AEM.00592-09.

Zhou X, Bent SJ, Schneider MG, Davis CC, Islam MR, and Forney LJ. 2004. Characterization of vaginal microbial communities in adult healthy women using cultivation-independent methods. Microbiology 150:2565-2573 DOI: 10.1099/mic.0.26905-0.

Zhou X, Hansmann MA, Davis CC, Suzuki H, Brown CJ, Schutte U, Pierson JD, and Forney LJ. 2010. The vaginal bacterial communities of Japanese women resemble those of women in 
515 other racial groups. FEMS Immunology and Medical Microbiology 58:169-181 DOI:

516 10.1111/j.1574-695X.2009.00618.x.

517

518 


\section{Figure 1}

The hierarchical clustering tree and the heat map of bacterial taxa from vaginal samples

The hierarchical clustering of 25 vaginal microbiota was generated based on the bacterial abundances of OTUs (A). The heat map showing the log2 of absolute read counts of the most abundant OTUs using the color key (B). The red-and blue bar below the tree indicates the lactobacilli-dominated and non-lactobacilli dominated group, respectively. 
A

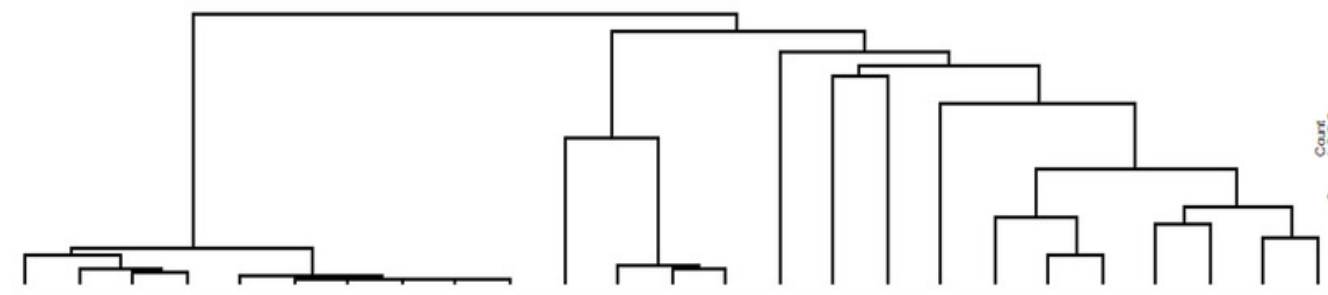

B

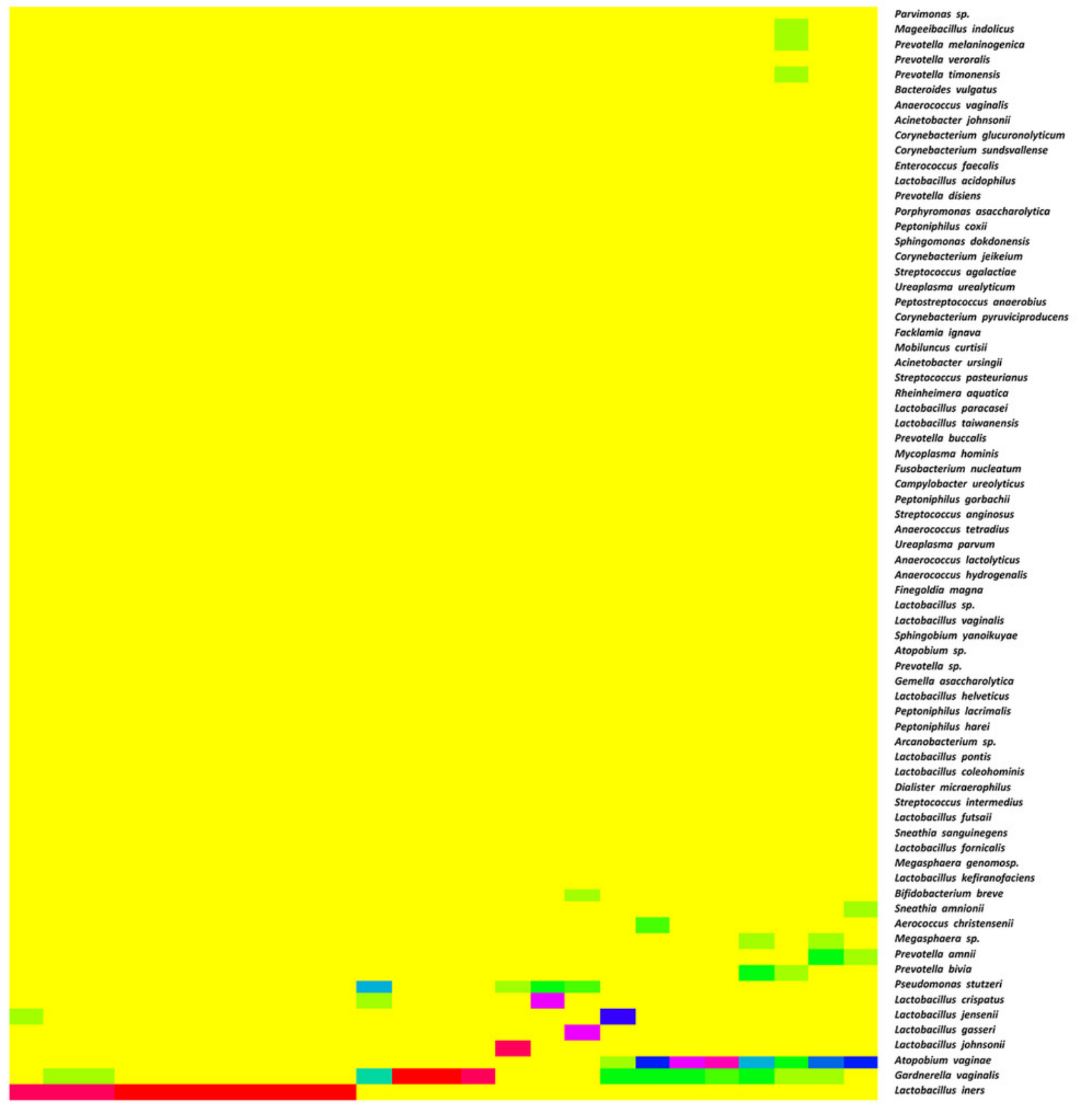


Figure 2

Shannon diversity index values in lactobacilli-dominated and non-lactobacilli-dominated groups

Shannon diversity index values indicating diversity of bacterial taxa in both groups, as shown using Tukey's boxplots

$P<0.001$

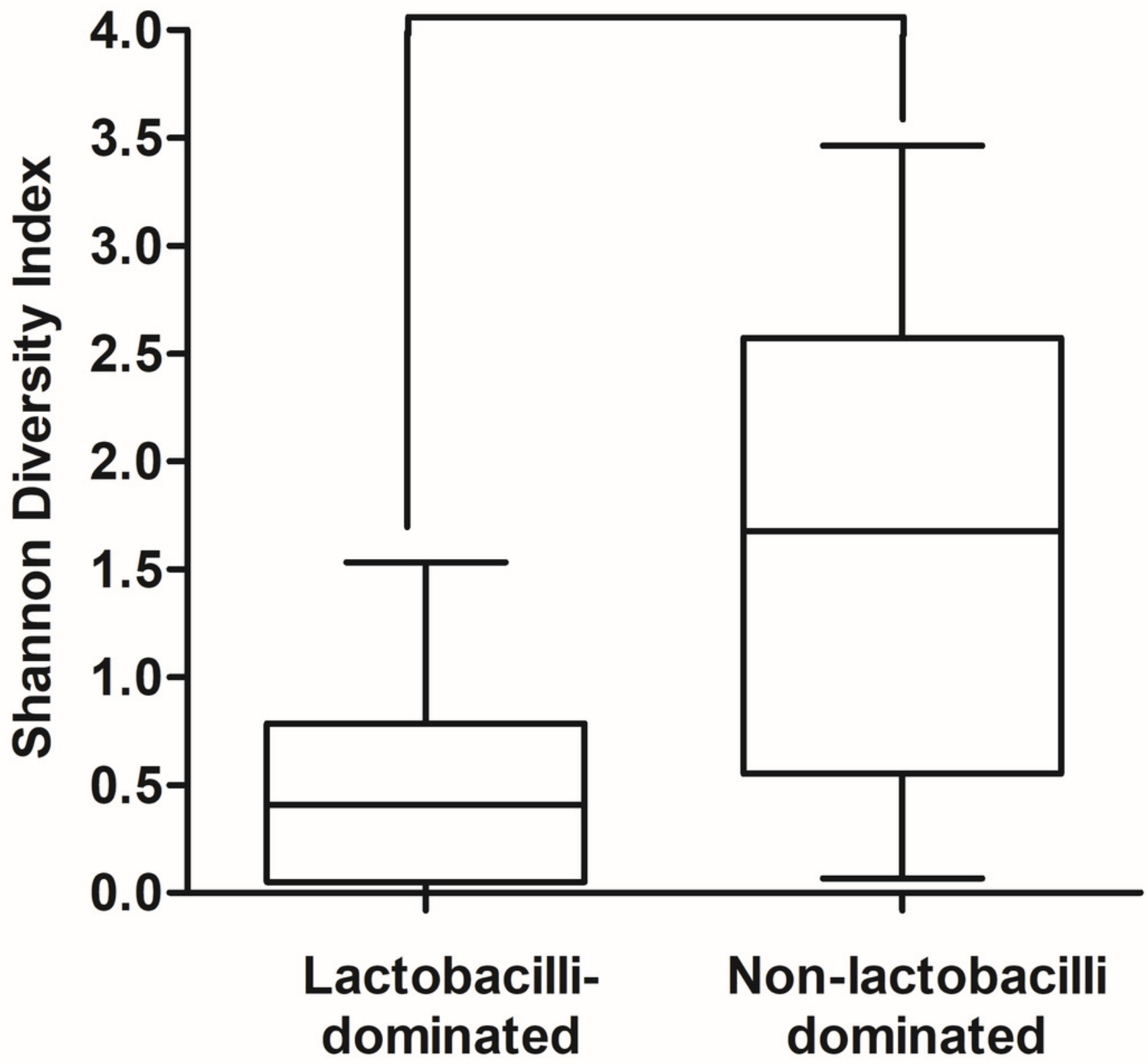


Figure 3

Rarefaction curves for observed species (OTUs) (A) and Chaol (B)

Both were used to estimate detected bacterial richness in lactobacilli-dominated and nonlactobacilli dominated groups.

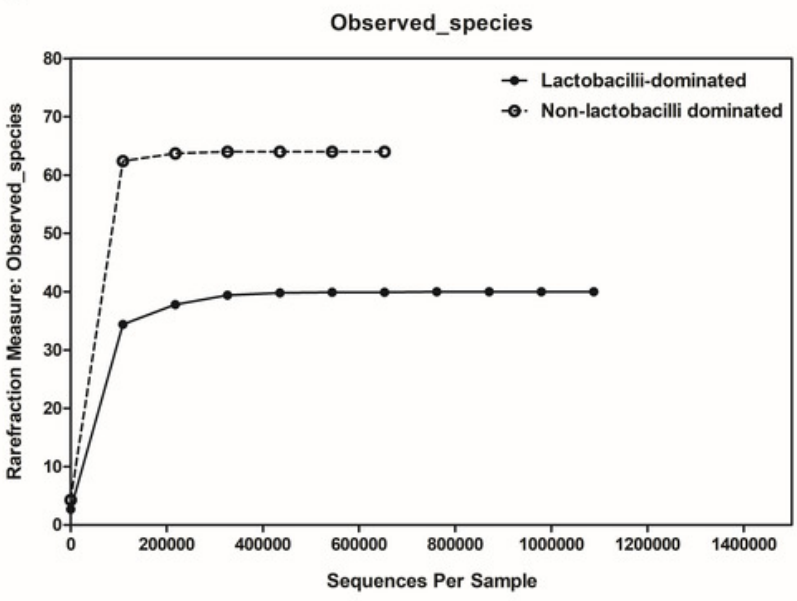

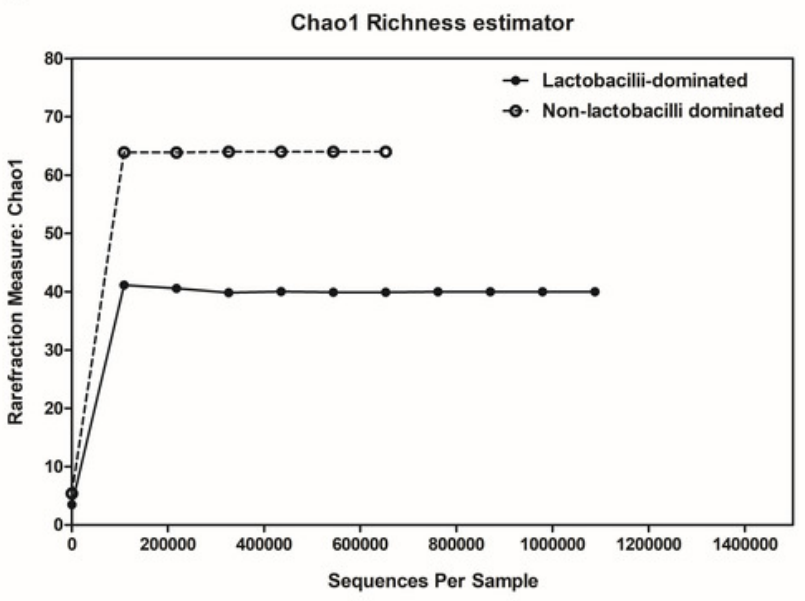




\section{Figure 4}

Principle coordinates analysis (PCoA) plot of Bray-Curtis dissimilarity indices among all samples.

- Lactobacilli-dominated

$\bigcirc$ Non-lactobacilli dominated

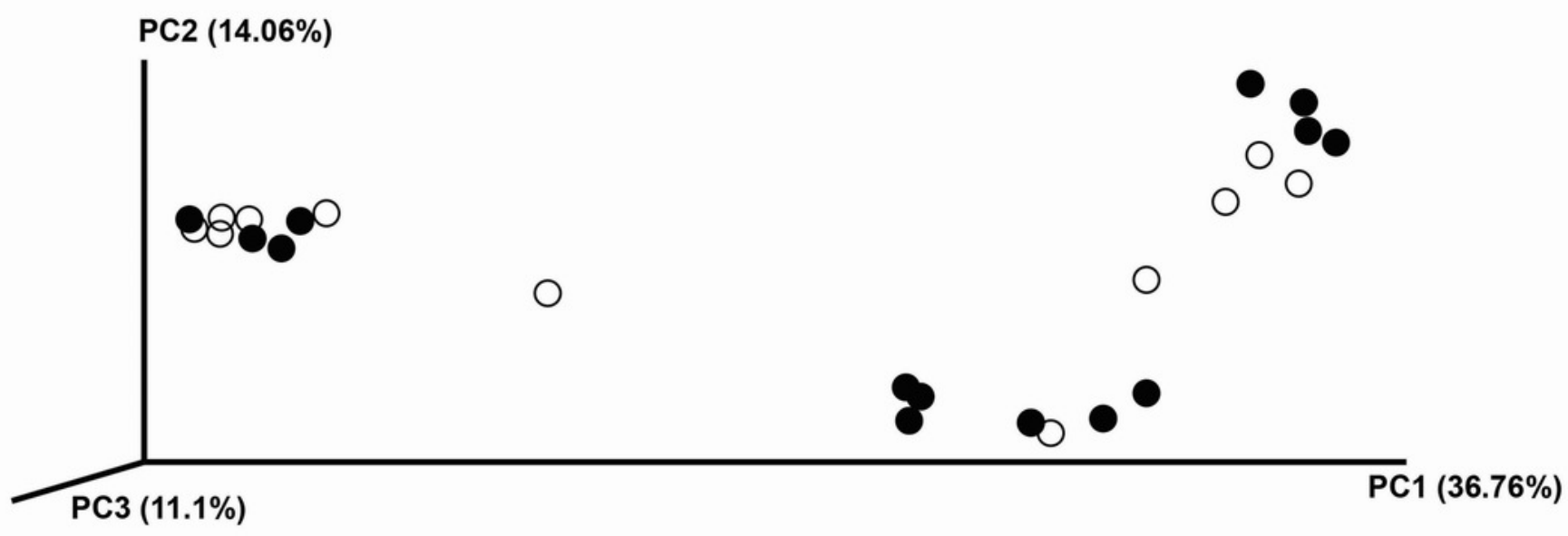


Figure 5

Venn diagram showing numbers of unique and shared OTUs (species) in lactobacillidominated and non-lactobacilli dominated groups.

\section{Lactobacilli-dominated Non-lactobacilli dominated}

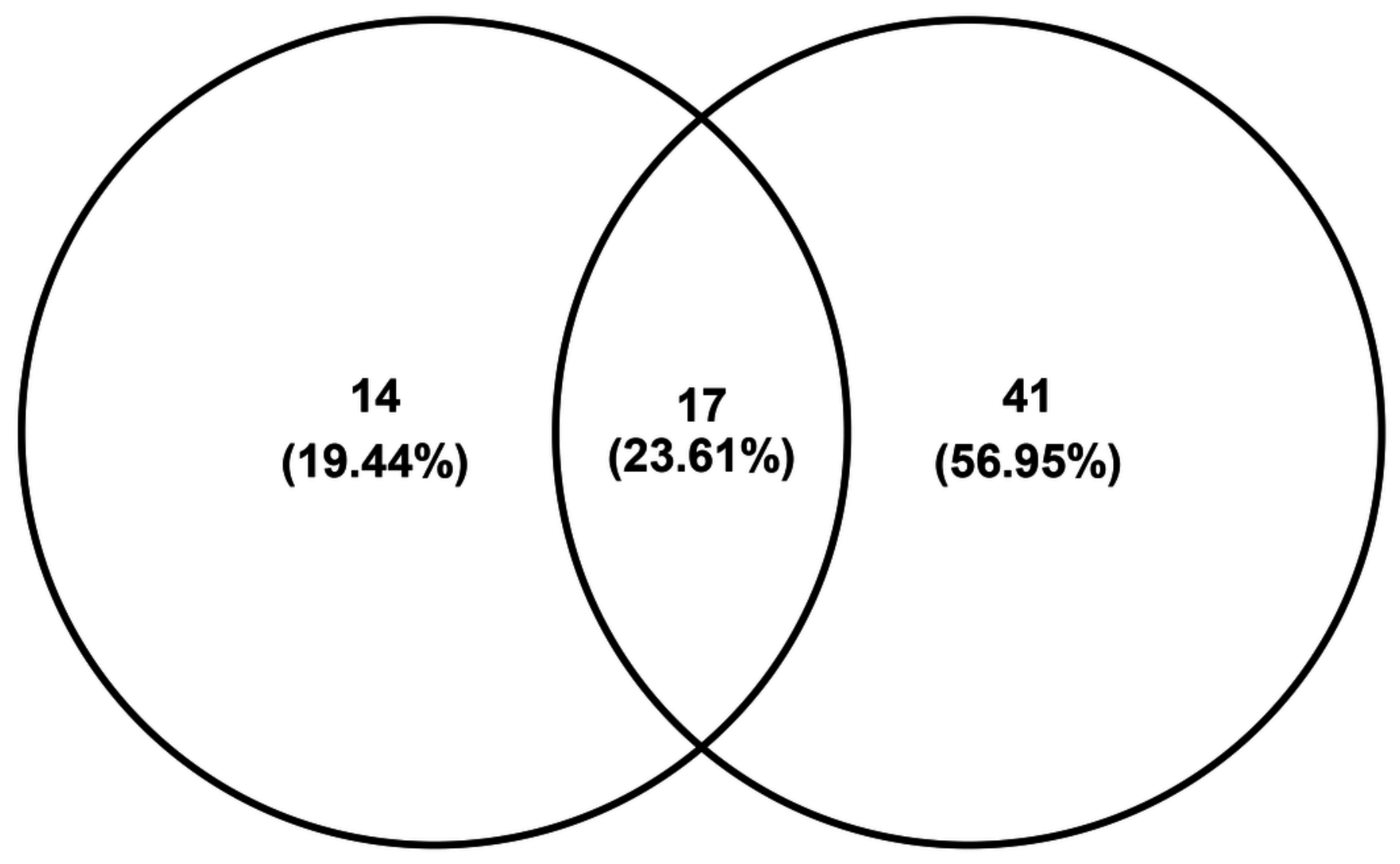


Figure 6

The most abundant bacterial species detected in vaginal samples in the lactobacillidominated and non-lactobacilli dominated groups.

Data are shown as mean with SEM. The bacterial species with abundance less than $1 \%$ are not shown. These include F. magna, L. kefiranofaciens, L. vaginalis, Peptoniphilus harei, Peptostreptococcus. anaerobius, S. intermedius and U. parvum . $*=$ significant difference.

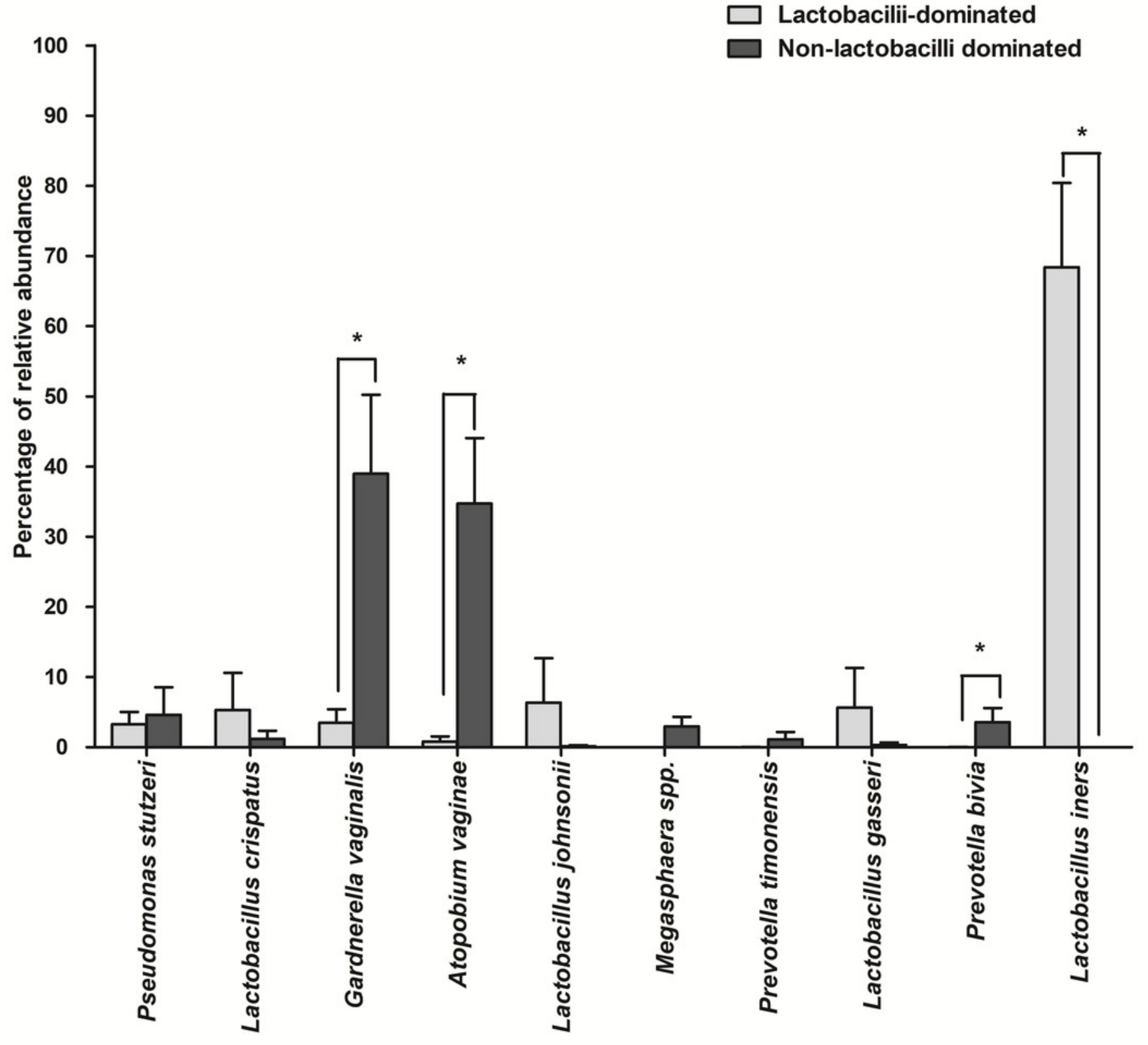




\section{Table $\mathbf{1}$ (on next page)}

Sociodemographic and behavioral characteristics, and health habits of the studied subjects. 
1 Table 1 Sociodemographic and behavioral characteristics, and health habits of the studied subjects.

\begin{tabular}{|c|c|c|c|}
\hline & $\begin{array}{l}\text { Lactobacilli- } \\
\text { dominated } \\
(N=14)\end{array}$ & $\begin{array}{l}\text { Non-lactobacilli- } \\
\text { dominated } \\
(\mathrm{N}=11)\end{array}$ & $\begin{array}{c}\text { Total } \\
(\mathrm{N}=\mathbf{2 5})\end{array}$ \\
\hline & n (\%) & n (\%) & n (\%) \\
\hline Median age (years) [range] & $40[31-45]$ & $33[27-45]$ & $39[27-45]$ \\
\hline Median BMI $\left(\mathrm{kg} / \mathrm{m}^{2}\right)$ [range] & $21.1[16.0-35.8]$ & $21.4[19.3-30.4]$ & $21.2[16.0-35.8]$ \\
\hline Higher vocational or university education & $12(85.7)$ & $10(90.9)$ & $22(88.0)$ \\
\hline Currently married & $11(78.6)$ & $10(90.9)$ & $21(84.0)$ \\
\hline$\geq 1$ child delivered & $10(71.4)$ & $6(54.5)$ & $16(64)$ \\
\hline Current smokers & $0(0)$ & $0(0)$ & $0(0)$ \\
\hline Income $<400$ USD per month & $4(28.6)$ & $5(45.5)$ & $9(36.0)$ \\
\hline Yogurt use $\geq 1$ time per week & $13(92.9)$ & $11(100.0)$ & $24(96.0)$ \\
\hline Alcohol consumption $\geq 1$ per week & $3(21.4)$ & $3(27.3)$ & $6(24.0)$ \\
\hline Exercise $\geq 1$ time per week & $12(85.7)$ & $8(72.7)$ & $20(80.0)$ \\
\hline Sexual activity $\geq 1$ time per week & $9(64.3)$ & $9(81.8)$ & $18(72.0)$ \\
\hline Sexual partner $>1$ time in previous year & $6(42.9)$ & $1(9.1)$ & $7(28.0)$ \\
\hline Regular ovulatory menstrual cycles in six months & $12(85.7)$ & $10(71.4)$ & $22(88.0)$ \\
\hline Current hormonal contraceptive use & $7(50.0)$ & $2(18.2)$ & $9(36.0)$ \\
\hline Vaginal douching done in previous month & $1(7.1)$ & $1(9.1)$ & $2(8.0)$ \\
\hline Abnormal discharge in previous month & $0(0)$ & $2(18.2)$ & $2(8.0)$ \\
\hline Female sterilization & $5(35.7)$ & $4(36.4)$ & $9(36.0)$ \\
\hline
\end{tabular}

2 Abbreviations: BMI = body mass index, USD = United States Dollar

3 All variables were self-reported.

4 Statistically not significant for any factor. $P$-values were calculated using chi-squared or Fisher's exact analysis for

5 assessment of association of frequency between groups and the Mann-Whitney U-Test for comparison of means. 